\title{
Starspot-Induced Radial Velocity Jitter During a Stellar Cycle
}

\author{
Heidi Korhonen ${ }^{1,2}$, Jan Marie Andersen ${ }^{2,3}$ and Silva Järvinen ${ }^{4}$ \\ ${ }^{1}$ Niels Bohr Institute, University of Copenhagen, Juliane Maries Vej 30, DK-2100 Copenhagen, \\ Denmark \\ ${ }^{2}$ Centre for Star and Planet Formation, Natural History Museum of Denmark, University of \\ Copenhagen, Øster Voldgade 5-7, DK-1350, Copenhagen, Denmark \\ ${ }^{3}$ Department of Astronomy, Boston University, 725 Commonwealth Avenue, Boston, MA \\ 02215, USA \\ ${ }^{4}$ Leibniz-Institut für Astrophysik Potsdam (AIP), An Der Sternwarte 16, D-14482 Potsdam, \\ Germany
}

\begin{abstract}
Late-type stars exhibit cool regions on their surface, the stellar equivalent of sunspots. These dark starspots can also mimic the radial velocity variations caused by orbiting planets, making it at times difficult to distinguish between planets and activity signatures. The amount of spots on the Sun and other cool stars changes cyclically during an activity cycle, which has length varying from about a year to longer than the solar 11 years. In this work we investigate the influence of varying amount of starspots on the sparsely sampled radial velocity observations - which are the norm in the radial velocity studies searching for exoplanets on wide orbits. We study two simulated cases: one with a random spot configuration, and one where the spot occurrence is concentrated. In addition we use Doppler images of young solar analogue V889 Her as a high activity case.
\end{abstract}

Keywords. stars: activity, atmospheres, planetary systems, rotation, spots

\section{Introduction}

In late-type stars (mainly spectral types $\mathrm{G}-\mathrm{M}$ ) magnetic fields often manifest themselves as cool areas on the stellar surface. These starspots can also show similar spectral effects as the stellar radial velocity caused by orbiting planets, introducing so-called radial velocity 'jitter', which at times can make it difficult to distinguish between planets and activity signatures (see, e.g., Queloz et al. 2001). Recent years several studies on these effects have been carried out, e.g. Saar \& Donahue (1997) and Boisse, Bonfils \& Santos (2012) on solar-like stars and Reiners et al. (2010) and Barnes, Jeffers \& Jones (2010) on M dwarfs. Still, no studies of the effect of the cyclic magnetic activity on sparse radial velocity datasets have been carried out.

The amount of spots on the Sun changes cyclically with the well-known 11 year cycle. Stars can have similar cycle lengths as the Sun (e.g., Baliunas et al. 1995) and all the way down to much shorter, 1-2 year cycles (e.g., Metcalfe et al. 2010). The radial velocity measurements for planet searches are often not continuous, and especially for planets on wide orbits the measurements will be obtained from different time points during the activity cycle. In this contribution we study the effects that has on radial velocity measurements. 

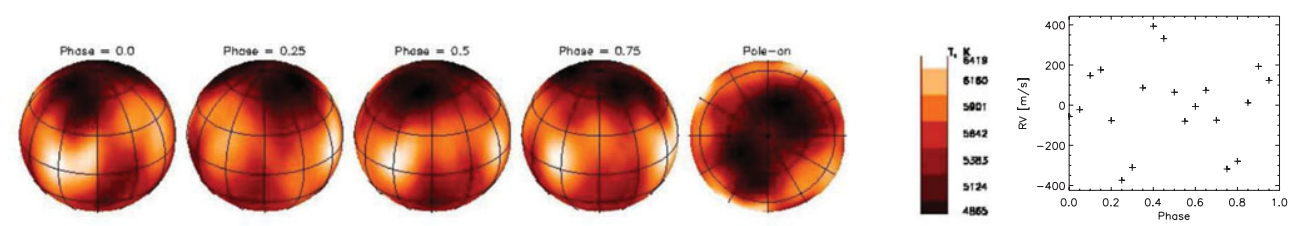

Figure 1. July/August 1999 temperature map of V889 Her from Järvinen et al. (2008) and corresponding radial velocity jitter.
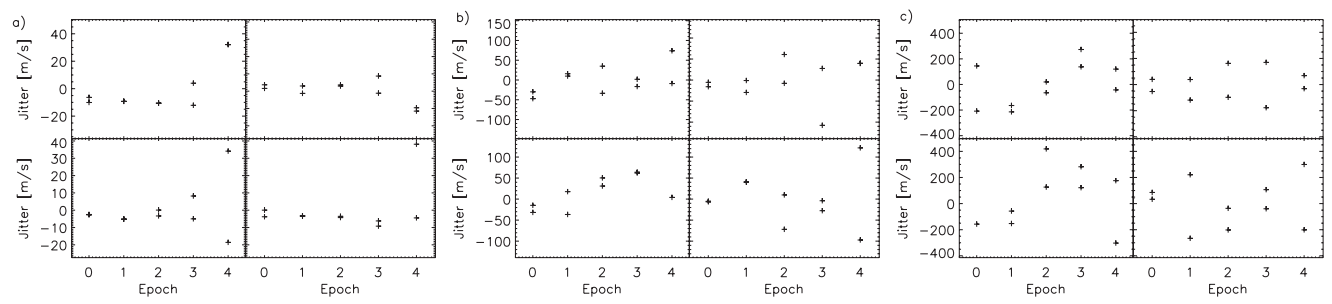

Figure 2. Radial velocity jitter during a stellar cycle. For all three cases, random spots (a), concentrated spots (b) and high activity (c), two randomly chosen longitudes from each surface distribution is plotted. For each case four randomly chosen sets are shown.

\section{Results}

We use the methods described by Andersen \& Korhonen (2014) for simulating spot patterns and calculating the jitter from them. For studying the jitter during the stellar activity cycle three different cases are used. For the simulated cases the total amount of spots on the surface is varied cyclically for two different spot configurations; one where the spots are completely randomly distributed in longitude and latitude, and another one where they are concentrated at longitudes 180-270 deg and around latitude $45 \mathrm{deg}$. The amount of spots is varied between five and fifty. The third case is a very high activity one, where five Doppler images of young solar analogue V889 Her are used from different epochs (Järvinen et al. 2008).

\subsection{Jitter from high activity young solar-like star}

V889 Her is a young, solar-like star, which can be considered as an analogue for the activity the young Sun could have exhibited. In Fig. 1 the surface spot distribution on V889 Her in July/August 1999 and the corresponding radial velocity jitter are shown as an example. The huge starspots seen on the surface of V889 Her naturally cause very large jitter.

\subsection{Jitter over stellar cycle}

For the simulated datasets where the number of spots is varied with the activity cycle, the jitter is determined over the full stellar rotation, at five different time points over half of the activity cycle - from minimum to maximum. From each time point two measurements are randomly selected to denote the radial velocities obtained during one observing run.

For all three cases (high activity case, simulated random spots and simulated concentrated spots) two random measurements are selected at each time point and plotted in Fig. 2. This mimics the observations for real radial velocity surveys where often only few data points are obtained at each epoch (i.e., observing run), and where the time difference between the epochs can be large. In all the cases studied here the randomly selected points can show patterns over half a stellar cycle (from minimum to maximum) that in some cases could be interpreted as a periodic signal. If hundred random jitter 
measurements are obtained the mean jitter is close to zero, but the standard deviation gets large, especially in the concentrated spot case.

\section{Conclusions}

We have investigated spot jitter from simulated spot configuration at different epochs with changing number of spots on the surface. The spot jitter over the cycle can in some cases cause small periodic signal, which could mimic planets on long period orbits. We also investigated jitter from a young, active, solar-like star, V889 Her. Jitter from the V889 Her Doppler images is naturally very large. Similarly the concentrated spot configurations introduce larger jitter amplitude than random spot configurations. The jitter from randomly distributed spots depends strongly on how the spots are exactly located. Even relatively small spots can introduce jitter which can drown the signal from an Earth-sized planet.

Acknowledgments. H.K. acknowledges the support from the European Commission under the Marie Curie IEF Programme in FP7, and an IAU travel grant to participate the General Assembly. J.M.A. acknowledges support through an NSF Graduate Research Fellowship and the Nordic Research Opportunity award, and travel grants from IAU and AAS.

\section{References}

Andersen, J. M. \& Korhonen, H. 2014, in Formation, Detection, and Characterization of Extrasolar Habitable Planets, Proceedings of the IAU Symposium 293, ed. N. Haghighipour (Cambridge University Press), 197

Baliunas, S. L., Donahue, R. A., Soon, W. H., et al. 1995, ApJ, 438, 269

Barnes, J. R., Jeffers, S. V., \& Jones, H. R. A. 2010, MNRAS 412, 1599

Boisse, I., Bonfils, X., \& Santos, N. C. 2012, A\&SA545, A109

Hackman, T., Jetsu, L., \& Tuominen, I. 2001, A\& $A$, 374, 171

Järvinen, S. P., Korhonen, H., Berdyugina, S. V., et al. 2008, A\& A 488, 1047

Kupka, F., Piskunov, N., Ryabchikova, T. A., Stempels, H. C., \& Weiss, W. W. 1999, A $\& A S$ 138,119

Kurucz, R. L. 1993, Kurucz CD No. 13

Metcalfe, T. S., Basu, S., Henry, T. J., et al. 2010, ApJL, 723, L213

Piskunov, N. E., Tuominen, I., \& Vilhu, O. 1990, A\&A 230, 363 ApJ, 723, 213

Queloz, D., Henry, G. W., Sivan, J. P., et al. 2001, A\&A 379, 279

Reiners, A., Bean, J. L., Huber, K. F., et al. 2010, ApJ 710, 432

Saar, S. H. \& Donahue, R. A. 1997, ApJ 485, 319 